\title{
PERTURBATION HYDROGEN-ATOM SPECTRUM IN A SPACE WITH THE LORENTZ-COVARIANT DEFORMED ALGEBRA WITH MINIMAL LENGTH
}

\author{
M. I. Samar, V. M. Tkachuk \\ Department for Theoretical Physics, Ivan Franko National University of Lviv \\ 12, Drahomanov St., Lviv, Ukraine
}

(Received June 26, 2009; received in final form November 25, 2009)

\begin{abstract}
We study energy spectrum of the hydrogen atom problem in the Dirac theory with the Lorentzcovariant deformed algebra leading to minimal length. Using conventional perturbation theory we calculate the correction to any energy level besides some "problem states" in a simple case of deformation when one deformation parameter vanishes.

Key words: deformed algebras, minimal length, Dirac equation, hydrogen atom.

PACS number(s): 02.40.Gh, 03.65.Pm, 31.15.Xp
\end{abstract}

\section{INTRODUCTION}

In recent years there has been a growing interest in the study of quantum mechanical systems with deformed commutation relations. It is motivated by several independent lines of theoretical physics (e.g., string theory and quantum gravity), which suggest the existence of minimal length as a finite lower bound to the possible resolution of length [1-3]. Kempf et al. showed that such an effect can be achieved by modifying usual canonical commutation relations $([4-7])$. The deformed commutation relation according to Kempf et al. in $D$-dimensional space reads

$$
\begin{aligned}
{\left[\hat{X}_{i}, \hat{P}_{j}\right] } & =i \hbar\left[\left(1+\beta \hat{P}^{2}\right) \delta_{i j}+\beta^{\prime} \hat{P}_{i} \hat{P}_{j}\right] \\
{\left[\hat{X}_{i}, \hat{X}_{j}\right] } & =i \hbar \frac{2 \beta-\beta^{\prime}+\left(2 \beta+\beta^{\prime}\right) \beta \hat{P}^{2}}{1+\beta \hat{P}^{2}}\left(\hat{P}_{i} \hat{X}_{j}-\hat{P}_{j} \hat{X}_{i}\right) \\
{\left[\hat{P}_{i}, \hat{P}_{j}\right] } & =0
\end{aligned}
$$

Here $\beta$ and $\beta^{\prime}$ are two small nonnegative parameters.

The proposed idea of a deformed commutation relation leading to minimal length was not new. As early as 1947 Snyder introduced the Lorentz-covariant quantized spacetime, dropping the usual hypothesis of commutativity of the coordinates [8]. Howewer, for a long time this idea attracted little attention of physicists. There have been few works on this subject in the last forty years [9-13]. An interest in this problem was revived with the investigations in quantum gravity and string theory.

It is worth mentioning that deformed algebra (1) is a nonrelativistic one, i. e. it violates the Lorentz symmetry. In paper [14] the $D$-dimensional two-parameter deformed algebra (1) was generalized to the $(D+1)$-dimensional quantized spacetime Lorentz-covariant one, which contains the Snyder algebra as a special case $(D=3, \beta=0)$

$$
\begin{aligned}
& {\left[\hat{X}^{\mu}, \hat{P}^{\nu}\right]=-i \hbar\left[\left(1-\beta \hat{P}_{\rho} \hat{P}^{\rho}\right) g^{\mu \nu}-\beta^{\prime} \hat{P}^{\mu} \hat{P}^{\nu}\right]} \\
& {\left[\hat{X}^{\mu}, \hat{X}^{\nu}\right]=i \hbar \frac{2 \beta-\beta^{\prime}-\left(2 \beta+\beta^{\prime}\right) \beta \hat{P}_{\rho} \hat{P}^{\rho}}{1-\beta \hat{P}_{\rho} \hat{P}^{\rho}}\left(\hat{P}^{\mu} \hat{X}^{\nu}-\hat{P}^{\nu} \hat{X}^{\mu}\right),} \\
& {\left[\hat{P}^{\mu}, \hat{P}^{\nu}\right]=0}
\end{aligned}
$$

with $g^{\mu \nu}=g_{\mu \nu}=\operatorname{diag}(1,-1,-1, \ldots,-1)$ being the metric tensor. Algebra (2) is an entirely new algebra, which cannot be reduced to algebra (1) in the nonrelativistic limit.

It is interesting to study the influence of minimal length assumption on the properties of quantum systems. Deformed commutation relations (1) were applied to the consideration of different quantum mechanical problems. For the review of the studied issues see [15]. In a space with the deformed Lorentz-covariant algebra (2), however, the exact solution of a $(1+1)$-dimensional Dirac oscillator in the simplest case of deformation, when one deformation parameter vanishes, is still the only problem that has been studied [14]. So this research area remains almost unexplored.

The hydrogen atom is a unique quantum mechanical system due to a highly accurate theoretical prediction and the most precise experimental data. Studies of this system may significantly contribute to our understanding of the fundamentals of the structure of the Universe. Studying the hydrogen atom in deformed space with minimal length we could come close to the answer whether the finite lower bound to the possible resolution of length does or does not exist. There are only a few papers on this theme in the case of Kempf's deformation [16-20].

Brau [16] considered the special case of deformation $\beta^{\prime}=2 \beta$ in which the position coordinates commute in linear approximation over the deformation parameters. In such simplest case the energy spectrum was calculated using perturbation theory. It was found that the deformation with minimal length decreases the depth of the hydrogen atom potential well. In [17] authors attempted to find the modifications to the energy of the zero angular momentum states, i.e. s-states, in momentum representation. Their results differ from the ones proposed in [16], namely the correction to the energy spectrum is opposite in sign. A general case of deformation, without any assumption on the deformation parameter, was considered in [18]. The perturbation hydrogen-atom spectrum was obtained analytically for all states besides $s$-states. To overcome the divergences which appeared in the calculation of the corrections to the $s$-level due to the term proportional to $1 / r^{3}$ the authors used a numerical method and cut-off procedure. It is worth mentioning 
that in a special case of deformation $\beta^{\prime}=2 \beta$ the results obtained by Benczik et al. reproduce those of Brau. A modified perturbation theory based on the shifted expansion of $\hat{R}^{-1}$ which does not contain the divergent terms was proposed in [19]. Such an idea gives a possibility to calculate the energy correction to any energy level including $s$-levels $[19,20]$.

In the present paper we develop the perturbation theory to calculate corrections to the energy spectrum of the hydrogen atom in space with a special case of deformation $\beta^{\prime}=0$ of the Lorentz-covariant commutation relations (2).

The paper is organized as follows. In Section II we discuss different representations of the Lorentz-covariant deformed algebra. In Section III we point out the solution of the relativistic hydrogen atom in ordinary quantum mechanics to introduce notations to be used in the paper. Considering the special case of deformation we obtain corrections to the energy spectrum of Coulomb problem in Dirac theory in Section IV using conventional perturbation theory. Finally, Section V contains the conclusion.

\section{REPRESENTATIONS OF THE ALGEBRA}

It is useful to represent the operators of position $\hat{X}^{\mu}$ and momenta $\hat{P}^{\mu}$ satisfying deformed algebra (2) by op- erators $\hat{x}^{\mu}$ and $\hat{p}^{\mu}$ which obey the canonical commutation relations

$$
\left[\hat{x}^{\mu}, \hat{p}^{\nu}\right]=-i \hbar g^{\mu \nu}
$$

The representation that leaves the operator of momenta undeformed reads

$$
\left\{\begin{array}{l}
\hat{X}^{\mu}=\left(1-\beta \hat{p}_{\rho} \hat{p}^{\rho}\right) \hat{x}^{\mu}-\beta^{\prime} \hat{p}^{\mu} \hat{p}_{\rho} \hat{x}^{\rho}+i \hbar \gamma p^{\mu} \\
\hat{P}^{\mu}=\hat{p}^{\mu}
\end{array}\right.
$$

where $\gamma$ is an arbitrary real constant which does not change the commutation relations. In momentum representation $\hat{x}^{\mu}=-i \hbar g^{\mu \nu} \partial / \partial p^{\nu}, \hat{p}^{\mu}=p^{\mu}$ we need to redefine the inner product by introducing the weight function.

$$
\begin{aligned}
\langle\psi \mid \phi\rangle & =\int \frac{d^{D} p}{\left[1-\left(\beta+\beta^{\prime}\right) p_{\nu} p^{\nu}\right]^{\alpha}} \psi^{*}\left(p^{\mu}\right) \phi\left(p^{\mu}\right), \\
\alpha & =\frac{2 \beta+\beta^{\prime}(D+2)-2 \gamma}{2\left(\beta+\beta^{\prime}\right)} .
\end{aligned}
$$

This provides the Hermiticity of the position operators. If we choose $\gamma=\beta+\beta^{\prime}(D+2) / 2$ the weight function reduces to 1 . At such an assumption about the value of $\gamma$ we can write down the position operators in the Hermitian form:

$$
\left\{\begin{array}{l}
\hat{X}^{\mu}=\hat{x}^{\mu}-\frac{\beta}{2}\left[\hat{p}_{\rho} \hat{p}^{\rho} \hat{x}^{\mu}+\hat{x}^{\mu} \hat{p}_{\rho} \hat{p}^{\rho}\right]-\frac{\beta^{\prime}}{2}\left[\hat{p}^{\mu} \hat{p}_{\rho} \hat{x}^{\rho}+\hat{x}_{\rho} \hat{p}^{\rho} \hat{p}^{\mu}\right] \\
\hat{P}^{\mu}=\hat{p}^{\mu}
\end{array}\right.
$$

Using the "pseudoposition" representation

$$
\left\{\begin{array}{l}
\hat{x}^{\mu}=x^{\mu} \\
\hat{p}^{\mu}=i \hbar g^{\mu \nu} \frac{\partial}{\partial x^{\nu}}
\end{array}\right.
$$

we calculate the square distance operator in the $(3+1)$ dimensional case $\hat{R}^{2}=\sum_{i=1}^{3}\left(\hat{X}^{i}\right)^{2}$ in the first order in $\beta, \beta^{\prime}$ :

$$
\begin{aligned}
R^{2} & =r^{2}+\left(\beta+\beta^{\prime}\right)\left[\hat{p}^{2} r^{2}+r^{2} \hat{p}^{2}\right]-2 \beta\left(\hat{p}^{0}\right)^{2} r^{2}+3 \hbar^{2} \beta \\
& -2 \beta^{\prime} \hat{L}^{2}-\frac{\beta^{\prime}}{2}\left(\hat{p}^{0} c t+c t \hat{p}^{0}\right)(\mathbf{r} \hat{\mathbf{p}}+\hat{\mathbf{p}} \mathbf{r})
\end{aligned}
$$

with $\hat{\mathbf{L}}$ being the angular momentum. An expansion of the inverse distance in the series over the parameters of deformation up to the first order reads

$$
\begin{aligned}
\hat{R}^{-1} & =\frac{1}{r}-\frac{\beta+\beta^{\prime}}{2}\left(\frac{1}{r} \hat{p}^{2}+\hat{p}^{2} \frac{1}{r}\right)+\beta\left(\hat{p}^{0}\right)^{2} \frac{1}{r}-\frac{2 \beta-\beta^{\prime}}{2} \frac{\hbar^{2}}{r^{3}} \\
& +\beta^{\prime} \frac{\hat{L}^{2}}{r^{3}}-\beta^{\prime} \frac{\left(\hat{p}^{0} c t+c t \hat{p}^{0}\right)}{4}\left(\frac{1}{r} \hat{p}^{2}-\hat{p}^{2} \frac{1}{r}\right) .
\end{aligned}
$$

Another representation that satisfies algebra (2) in the first order in $\beta, \beta^{\prime}$ in contrast to exact representation (7) reads

$$
\left\{\begin{array}{l}
\hat{X}^{\mu}=\hat{x}^{\mu}-\frac{2 \beta-\beta^{\prime}}{4}\left(\hat{x}^{\mu} \hat{p}_{\rho} \hat{p}^{\rho}+\hat{p}_{\rho} \hat{p}^{\rho} \hat{x}^{\mu}\right), \\
\hat{P}^{\mu}=\hat{p}^{\mu}-\frac{\beta^{\prime}}{2} \hat{p}^{\mu} \hat{p}_{\rho} \hat{p}^{\rho} .
\end{array}\right.
$$

By analogy, the square distance operator and inverse distance operator in representation $(11),(8)$ has the form:

$$
\begin{aligned}
R^{2} & =r^{2}+\frac{\left(2 \beta-\beta^{\prime}\right)}{2}\left(\left(\hat{p}^{2}-\left(\hat{p}^{0}\right)^{2}\right) r^{2}\right. \\
& \left.+r^{2}\left(\hat{p}^{2}-\left(\hat{p}^{0}\right)^{2}\right)+3 \hbar^{2}\right), \\
\hat{R}^{-1} & =\frac{1}{r}-\frac{t\left(2 \beta-\beta^{\prime}\right)}{4}\left(\frac{1}{r}\left(\hat{p}^{2}-\left(\hat{p}^{0}\right)^{2}\right)\right. \\
& \left.+\left(\hat{p}^{2}-\left(\hat{p}^{0}\right)^{2}\right) \frac{1}{r}+\frac{2 \hbar^{2}}{r^{3}}\right) .
\end{aligned}
$$

Representations (7) and (11) coincide when $\beta^{\prime}=0$. 


\section{HYDROGEN ATOM IN THE DIRAC THEORY}

To review the notations to be used in this paper let us recall the solution of hydrogen atom problem in the Dirac theory with standart nondeformed algebra.

We write the Dirac Hamiltonian with a central force potential in the form

$$
\hat{H}=c \hat{\rho}_{a} \hat{P}+m c^{2} \hat{\rho}_{c}+U(r),
$$

where

$$
\hat{P}=\hat{\sigma}_{x} \hat{p}^{x}+\hat{\sigma}_{y} \hat{p}^{y}+\hat{\sigma}_{z} \hat{p}^{z}
$$

The choice of matrices $\sigma_{i}, i=x, y, z ; \rho_{j}, j=a, b, c$ can be performed in different ways. The following representation of these matrices is more convenient in some relations.

$$
\begin{aligned}
\hat{\sigma}_{x}=\left(\begin{array}{cc}
\hat{\sigma}_{x}^{0} & 0 \\
0 & -\hat{\sigma}_{x}^{0}
\end{array}\right), & \hat{\sigma}_{y}=\left(\begin{array}{cc}
\hat{\sigma}_{y}^{0} & 0 \\
0 & \hat{\sigma}_{y}^{0}
\end{array}\right), \\
\hat{\sigma}_{z}=\left(\begin{array}{cc}
\hat{\sigma}_{z}^{0} & 0 \\
0 & -\hat{\sigma}_{z}^{0}
\end{array}\right), & \\
\hat{\rho}_{a}=\left(\begin{array}{cc}
I & 0 \\
0 & -I
\end{array}\right), & \hat{\rho}_{b}=\left(\begin{array}{cc}
0 & \hat{\sigma}_{y}^{0} \\
\hat{\sigma}_{y}^{0} & 0
\end{array}\right), \\
\hat{\rho}_{c} & =\left(\begin{array}{cc}
0 & -i \hat{\sigma}_{y}^{0} \\
i \hat{\sigma}_{y}^{0} & 0
\end{array}\right) .
\end{aligned}
$$

Here $\hat{\sigma}_{x}^{0}, \hat{\sigma}_{y}^{0}, \hat{\sigma}_{z}^{0}$ denote standart Pauli spin matrices.

Following Fock [21], let us perform the transformation of equation

$$
\left[c \hat{\rho}_{a} \hat{P}+m c^{2} \hat{\rho}_{c}+U(r)\right] \psi=\hat{p}^{0} c \psi
$$

to spherical coordinates accompanied with canonical transformations. Any operator and wave function transform:

$$
\begin{aligned}
& \hat{A} \rightarrow \hat{A}^{*}=r \sqrt{\sin (\theta)} U \hat{A} U^{-1} \frac{1}{r \sqrt{\sin (\theta)}}, \\
& \psi \rightarrow \psi^{*}=r \sqrt{\sin (\theta)} U \psi,
\end{aligned}
$$

with $U=\left(\hat{I} \cos \frac{\theta}{2}+i \hat{\sigma}_{y} \sin \frac{\theta}{2}\right)\left(\hat{I} \cos \frac{\varphi}{2}+i \hat{\sigma}_{z} \sin \frac{\varphi}{2}\right)$ being the unitary transformation. Turning into stationary equation, we obtain

$$
\left[c \hat{\rho}_{a} \hat{P}^{*}+m c^{2} \hat{\rho}_{c}+U(r)\right] \psi^{*}=E^{0} \psi^{*}
$$

The solution of (21) for $\psi^{*}=\left(\psi_{1}^{*}, \psi_{2}^{*}, \psi_{3}^{*}, \psi_{4}^{*}\right)^{T}$ reads

$$
\begin{array}{ll}
\psi_{1}^{*}=f(r) Y(\theta, \varphi), & \psi_{2}^{*}=g(r) Z(\theta, \varphi), \\
\psi_{3}^{*}=f(r) Z(\theta, \varphi), & \psi_{4}^{*}=-g(r) Y(\theta, \varphi),
\end{array}
$$

with normalization conditions

$$
\begin{aligned}
& \int_{0}^{\infty} d r\left(|f|^{2}+|g|^{2}\right)=1 \\
& \int_{0}^{\pi} d \theta \int_{0}^{2 \pi} d \varphi\left(|Y|^{2}+|Z|^{2}\right)=1 .
\end{aligned}
$$

Here $Y(\theta, \varphi), Z(\theta, \varphi)$ are the so-called spherical functions. The functions $f(r)$ and $g(r)$ correspond to the form of the potential $U(r)$. For the Coulomb potential $U(r)=-\frac{e^{2}}{r}$

$$
\begin{aligned}
& f(r)=\frac{1}{\sqrt{2}}\left[\frac{F_{p k}\left(\frac{2 r}{a n^{*}}\right)-G_{p k}\left(\frac{2 r}{a n^{*}}\right)}{2 \sin \frac{\varepsilon}{2}}+i \frac{F_{p k}\left(\frac{2 r}{a n^{*}}\right)+G_{p k}\left(\frac{2 r}{a n^{*}}\right)}{2 \cos \frac{\varepsilon}{2}}\right], \\
& g(r)=\frac{1}{\sqrt{2}}\left[\frac{F_{p k}\left(\frac{2 r}{a n^{*}}\right)-G_{p k}\left(\frac{2 r}{a n^{*}}\right)}{2 \sin \frac{\varepsilon}{2}}-i \frac{F_{p k}\left(\frac{2 r}{a n^{*}}\right)+G_{p k}\left(\frac{2 r}{a n^{*}}\right)}{2 \cos \frac{\varepsilon}{2}}\right], \\
& F_{p k}(x)=C(p, k) x^{\frac{s}{2}} e^{-\frac{x}{2}} Q_{p}^{s}(x), \\
& G_{p k}(x)=C(p, k)\left(n^{*}-k\right) x^{\frac{s}{2}} e^{-\frac{x}{2}} Q_{p-1}^{s}(x) .
\end{aligned}
$$

Here $Q_{p}^{s}(x)=\frac{\Gamma(s+p+1)}{\Gamma(s+1)}{ }_{1} F_{1}(-p, s+1, x)$ are the Laguerre polynomial; $p=0,1,2, \ldots ; k$ is connected with the total angular momentum quantum number by $j=$ $|k|-\frac{1}{2}, k= \pm 1, \pm 2, \pm 3, \ldots ; C(p, k)=\sqrt{\frac{\left(n^{*}+k\right)}{p ! \Gamma(p+s+1)} \frac{\alpha^{2}}{n^{* 4}}}$ is the normalization constant; $\alpha$ denotes the fine structure constant; $a=\frac{\hbar^{2}}{m e^{2}}$ is the Bohr radius; $\varepsilon$ depends on energy $E^{0}$ by the following equation

$$
E^{0}=m c^{2} \cos \varepsilon .
$$

Quantum numbers which appear in formulas (25) are connected by

$$
s=2 \sqrt{k^{2}-\alpha^{2}}, \quad p+s / 2=\alpha \cot \varepsilon .
$$

We also denote $\frac{\alpha}{\sin \varepsilon}$ by $n^{*}$ due to a slight difference between this ratio and integer main quantum number $n=p+|k|$. Finally, the energy spectrum depending on quantum numbers $p$ and $k$ is

$$
E_{p k}^{0}=m c^{2} \frac{p+\sqrt{k^{2}-\alpha^{2}}}{\sqrt{\left(p+\sqrt{k^{2}-\alpha^{2}}\right)^{2}+\alpha^{2}}}
$$




\section{PERTURBATION OF THE ENERGY SPECTRUM}

In this section we calculate the correction to the energy spectrum of hydrogen atom in Dirac theory with the Lorentz-covariant deformed algebra with minimal length using conventional perturbation theory.

We study the Dirac equation in a $(3+1)$-dimensional case in the form of

$$
\left[c \hat{\rho}_{a}\left(\hat{\sigma}_{x} \hat{P}^{x}+\hat{\sigma}_{y} \hat{P}^{y}+\hat{\sigma}_{z} \hat{P}^{z}\right)+m c^{2} \hat{\rho}_{c}-\frac{e^{2}}{R}\right] \psi=\hat{P}^{0} c \psi,
$$

where operators of position $\hat{X}^{\mu}$ and momenta $\hat{P}^{\mu}$ satisfy deformed comutation relations (2).

As we saw in Section II, operators $\hat{P}^{0}$ and $\hat{R}^{-1}$ do not commute any more. That is why we would find no function to be the eigenfunction of the operator $\hat{P}^{0}$ and $\hat{H}$ simultaneously in contrast to the undeformed case. It is unknown for the authors how to write correctly stationary equation under such circumstances. Therefore we consider the case when one of the deforming parameters vanishes and the other one is positive, namely $\beta^{\prime}=0$ and $\beta>0$. This assumption on the deformation parameters guarantees commutativity of the operators $\hat{P}^{0}$ and $\hat{R}^{-1}$. Note that at such an assumption for the parameters of deformation representations (7) and (11) coincide.

We rewrite equation (29) taking into account only the first-order term in $\beta$

$$
\left(\hat{H}_{0}+\hat{V}_{\beta}\right) \psi=\hat{p}^{0} c \psi
$$

The Hamiltonian of the unperturbed problem reads

$$
\hat{H}_{0}=c \hat{\rho}_{a} \hat{P}+m c^{2} \hat{\rho}_{c}-\frac{e^{2}}{r},
$$

and the perturbation operator can be written as follows

$$
\hat{V}_{\beta}=\frac{\beta}{2} e^{2}\left(\left(\hat{p}^{2}-\left(\hat{p}^{0}\right)^{2}\right) \frac{1}{r}+\frac{1}{r}\left(\hat{p}^{2}-\left(\hat{p}^{0}\right)^{2}\right)+\frac{2 \hbar^{2}}{r^{3}}\right) .
$$

We perform a conversion to the stationary equation by assuming

$$
\psi(\mathbf{r}, t)=e^{-\frac{i}{\hbar} E t} \psi(\mathbf{r})
$$

Similarly to the undeformed case, we perform a transformation to spherical coordinates together with canonical transformations. Finally we have

$$
\left(\hat{H}_{0}^{*}+\hat{V}_{\beta}^{*}\right) \psi^{*}=E \psi^{*}
$$

with

$$
\begin{aligned}
& \hat{H}_{0}^{*}=c \hat{\rho}_{a} \hat{P}^{*}+m c^{2} \hat{\rho}_{c}-\frac{e^{2}}{r} \\
& \hat{V}_{\beta}^{*}=\frac{\beta}{2} e^{2}\left(\left(\left(\hat{p}^{*}\right)^{2}-\left(p^{0}\right)^{2}\right) \frac{1}{r}+\frac{1}{r}\left(\left(\hat{p}^{*}\right)^{2}-\left(p^{0}\right)^{2}\right)+\frac{2 \hbar^{2}}{r^{3}}\right)
\end{aligned}
$$

and

$$
\begin{aligned}
& \left(\hat{p}^{*}\right)^{2}=\left(\hat{P}^{*}\right)^{2}=\hat{p}_{r}^{2}-\frac{\hbar \hat{M}^{*}}{r^{2}}+\frac{\left(\hat{M}^{*}\right)^{2}}{r^{2}}, \\
& \hat{M}^{*} \psi^{*}=\hbar k \rho_{c} \psi^{*} \\
& p^{0}=\frac{E^{0}}{c} .
\end{aligned}
$$

Now we can calculate the corrections to the spectrum using the eigenfunctions of an undeformed relativistic hydrogen atom

$$
\begin{gathered}
\Delta E_{p k}^{(1)}=m c^{2} \frac{\hbar^{2} \beta}{a^{2}}\left(\frac{12 \alpha^{2}(2 p+s)\left(2 n^{*} k\left(\alpha^{2}+1\right)+k^{4}(2 p+s)\right)}{s\left(s^{2}-1\right)\left(s^{2}-4\right) n^{* 5}}+\right. \\
\left.+\frac{\alpha^{2}\left(-3 n^{* 2}+4 n^{*} k(2 p+s)-4 k^{2} \alpha^{2}\right)}{s\left(s^{2}-1\right) n^{* 5}}+\frac{\alpha^{2}-n^{* 2}+p^{2}-k^{2}}{s n^{* 3}}\right) .
\end{gathered}
$$


The correction to the energy spectrum $\Delta E_{p k}^{(1)}$ depends on two quantum numbers $p$ and $k$ connected with the usual main quantum number $n$ and the total angular momentum quantum number $j$ by

$$
n=p+|k|, \quad j=|k|-\frac{1}{2} .
$$

Formula (40) works for any allowed values for $p$ and $k$, besides $k= \pm 1$. For the states with such values of quantum number $k$ we obtain a divergent contribution of terms proportional to $\frac{1}{r}\left(\hat{p}^{*}\right)^{2}+\left(\hat{p}^{*}\right)^{2} \frac{1}{r}$ and $\frac{1}{r^{3}}$ to the energy correction. For example, for the ground state we have

$$
\left\langle\frac{1}{r^{3}}\right\rangle \sim \int_{0}^{\infty} d x x^{s-3} e^{-x}=\infty,
$$

because $s=2 \sqrt{1-\alpha^{2}}$ is less than 2 . The situation is rather similar to the nonrelativistic case of deformation and calls for a modification of the perturbation theory [19].

It is worth mentioning that if we omit the contribution of the term $\frac{\left(p^{0}\right)^{2}}{r}$ to $\Delta E_{p k}^{(1)}$ we will find a correction to the energy spectrum of a hydrogen atom in the Dirac theory with Kempf's deformation. In the nonrelativistic limit of $c \rightarrow \infty$ this correction as expected coincides with the result calculated in [18].

\section{CONCLUSION}

We studied the hydrogen atom problem in a space with the Lorentz-covariant deformed algebra with the assumption fo the deformation parameters $\beta \neq 0, \beta^{\prime}=0$. Using conventional perturbation theory we calculate the correction to the energy spectrum for all states besides some "problem states" with $|k|=1$. The difficulties are due to the terms proportional to $1 / r^{3}$ and $\frac{1}{r} \hat{p}^{2}+\hat{p}^{2} \frac{1}{r}$ contained in the perturbation operator because of their divergent contribution to the energy correction. The same problem with divergences appeared in the nonrelativistic case of deformation when one attempts to calculate the correction to $s$-levels of a hydrogen atom $[18,19]$.

One of the interesting problems is to extend the method over the case of a two-parameter deformed algebra. The authors expect that this problem to be the subject of one of our papers in the future.

\section{ACKNOWLEDGEMENT}

The authors thank Dr. Andrij Rovenchak and Dr. Mykola Stetsko who kindly read the manuscript.
[1] D. J. Gross, P. F. Mende, Nucl. Phys. B 303, 407 (1988).

[2] M. Maggiore, Phys. Lett. B 304, 65 (1993).

[3] E. Witten, Phys. Today 49, 24 (1996).

[4] A. Kempf, J. Math. Phys. 35, 4483 (1994).

[5] A. Kempf, G. Mangano, R. B. Mann, Phys. Rev. D 52, 1108 (1995).

[6] H. Hinrichsen, A Kempf, J. Math. Phys. 37, 2121 (1996).

[7] A. Kempf, J. Phys. A: Math. Gen. 30, 2093 (1997).

[8] H. S. Snyder, Phys. Rev. 71, 38 (1947).

[9] H. S. Snyder, Phys. Rev. 72, 68 (1947).

[10] C. N. Yang, Phys. Rev. 72, 874 (1947).

[11] E. J. Hellund, K. Tanaka, Phys. Rev. 94, 192 (1954).

[12] E. Fischbach, Phys. Rev. B 137, 642 (1965).

[13] M. R. Hamilton, G. Sandri, Phys. Rev. D 8, 1788 (1973).
[14] C. Quesne, V. M. Tkachuk, J. Phys. A 39, 10909 ( 2006).

[15] T. Fityo, Phys. Lett. A 372, 5872 (2008). T. V. Fityo, I. O. Vakarchuk, V. M. Tkachuk, J. Phys. A: Math. Gen. 39, 379 (2006).

[16] F. Brau, J. Phys. A 32, 7691 (1999).

[17] R. Akhoury, Y-P. Yao, Phys. Lett. B 572, 37 (2003).

[18] S. Benczik, L. N. Chang, D. Minic, T. Takeuchi, Phys. Rev. A 72, 012104 (2005).

[19] M. M. Stetsko, V. M. Tkachuk, Phys. Rev. A 74, 012101 (2006).

[20] M. M. Stetsko, Phys. Rev. A 74, 062105 (2006).

[21] V. A. Fock, Fundamentals of Quantum mechanics (Mir Publishers, Moscow, 1983) [Translation from the 2-nd edition].

\title{
ПЕРТУРБАЦІЙНИЙ СПЕКТР АТОМА ВОДНЮ У ПРОСТОРІ З ЛОРЕНЦ-КОВАРІАНТНОЮ ДЕФОРМОВАНОЮ АЛГЕБРОЮ ГАЙЗЕНБЕРГА 3 МІНІМАЛЬНОЮ ДОВЖИНОЮ
}

\author{
M. I. Самар, В. М. Ткачук \\ Кафедра теоретичної фізики Лъвівсъкого національного університету імені Івана Франка, \\ вул. Драгоманова, 12, Лъвів, 79005, Україна
}

\footnotetext{
Ми вивчаємо енерґетичний спектр атома водню в теорії Дірака з Лоренц-коваріантною деформованою алгеброю, що веде до мінімальної довжини. У простому випадку деформації, коли один із ї̈ параметрів дорівнює нулеві, ми, використовуючи звичайну теорію збурень, обчислили поправки до всіх енергетичних рівнів, крім деяких "проблемних" станів.
} 